Engineering and Design

Elsevier Editorial System(tm) for Fusion

Manuscript Draft

Manuscript Number:

Title: Structural analysis of the W7-X cryogenic pipe system

Article Type: Soft 25th Special Issue Paper

Keywords: cryogenic pipe system, structural analysis, supports, clash analysis

Corresponding Author: Mr. André Duebner,

Corresponding Author's Institution: Max-Planck-Institut für Plasmaphysik

First Author: André Duebner

Order of Authors: André Duebner; Daniel Zacharias; Victor Bykov; Felix Schauer; Michael Nagel; Marko Ihrke

Abstract: Wendelstein 7-X (W7-X), a modular advanced stellarator, is presently under construction at the Max-Planck-Institut für Plasmaphysik in Greifswald. Its magnet system (MS) consists of 50 non-planar and 20 planar superconducting coils which are arranged in five identical modules. The cryogenic cooling pipe system (CPS) for the MS is one of the remaining major components under manufacture. Since it is the easiest to adapt, its design and structural analysis had to be postponed until the design of the main MS components like coils, structure, and bus-bar system, influencing the layout and mechanical behaviour of the piping, had been finalized. The complex 3-dimensional routing of the cooling pipes is governed by avoiding clashes with the structure during cool-down and operation. Most of the pipe supports allow sliding and limited rotation in order to reduce stress levels, forces and moments in the pipes and supports. This given fact, in combination with high deformations of the supporting magnet system, makes the CPS behaviour highly non-linear. Furthermore, several connected components such as valves and coil terminals cannot take any significant loads. As a consequence, the transmission of forces and movements through these interfaces has to be limited by kinematic separation, using flexible hoses and compensators. The non-linear finite element (FE) model created for the first MS module to be assemled includes 159 pipe sections with a total length of $470 \mathrm{~m}$. The system encompasses approx. 80 flexible hoses and compensators, as well as 136 supports with clamps interconnecting up to 6 neighbouring lines. This paper presents the strategy of the CPS analysis, the FE model, as well as the various boundary conditions and limitations due to design requirements. The results of this most challenging parametric study are discussed, including variations of hose and compensator flexibilities as well as of the support gap sizes. 
Greifswald, $08^{\text {th }}$ August 2008

Dear Sir, Dear Madam,

Please find attached our paper for the SOFT 2008 entitled:

"Structural analysis of the W7-X cryogenic pipe system"

Coauthors:

Daniel Zacharias, Felix Schauer, Victor Bykov, Michael Nagel, Marko Ihrke

Thank you very much for considering our paper.

Yours sincerely,

André Dübner

Max-Planck-Institut für Plasmaphysik

Teilinstitut Greifswald

Wendelsteinstraße 1

17491 Greifswald

Germany

Tel: +49 - (0) 3834 / 882414

E-Mail: andre.duebner@ipp.mpg.de 


\title{
Structural analysis of the W7-X cryogenic pipe system
}

\author{
A. Dübner*, D. Zacharias, M. Nagel, V. Bykov, F. Schauer, M. Ihrke \\ Max-Planck-Institut für Plasmaphysik, Teilinstitut Greifswald, Member of the Euratom Association, \\ Wendelsteinstraße 1, 17491 Greifswald, Germany \\ * Correspondong author: Tel.: +49 383488 2414; fax +49 3834882439. \\ E-mail address: andre.duebner@ipp.mpg.de (A. Dübner)
}

\begin{abstract}
Wendelstein 7-X (W7-X), a modular advanced stellarator, is presently under construction at the Max-Planck-Institut für Plasmaphysik in Greifswald. Its magnet system (MS) consists of 50 nonplanar and 20 planar superconducting coils which are arranged in five identical modules. The cryogenic cooling pipe system (CPS) for the MS is one of the remaining major components under manufacture. Since it is the easiest to adapt, its design and structural analysis had to be postponed until the design of the main MS components like coils, structure, and bus-bar system, influencing the layout and mechanical behaviour of the piping, had been finalized. The complex 3-dimensional routing of the cooling pipes is governed by avoiding clashes with the structure during cool-down and operation. Most of the pipe supports allow sliding and limited rotation in order to reduce stress levels, forces and moments in the pipes and supports. This given fact, in combination with high deformations of the supporting magnet system, makes the CPS behaviour highly non-linear. Furthermore, several connected components such as valves and coil terminals cannot take any significant loads. As a consequence, the transmission of forces and movements through these interfaces has to be limited by kinematic separation, using flexible hoses and compensators. The non-linear finite element (FE) model created for the first MS module to be assemled includes 159 pipe sections with a total length of $470 \mathrm{~m}$. The system encompasses approx. 80 flexible hoses and compensators, as well as 136 supports with clamps interconnecting up to 6 neighbouring lines. This paper presents the strategy of the CPS analysis, the FE model, as well as the various boundary conditions and limitations due to design requirements. The results of this most challenging
\end{abstract}


parametric study are discussed, including variations of hose and compensator flexibilities as well as of the support gap sizes.

\section{Introduction and overview}

Five basic cooling loops are required to supply the cryogenic component groups within the W7-X cryostat with cold helium: Cable-in-conduit conductors $(\approx 4 \mathrm{~K})$, coil casings, $(\approx 4 \mathrm{~K})$, magnet system central support structure $(\approx 4 \mathrm{~K})$, current leads $(\approx 4 \mathrm{~K}, \approx 50 \mathrm{~K})$, and thermal shields $(50 \mathrm{~K}-70 \mathrm{~K})$. In addition to that, safety exhaust pipes are necessary. Approximately 160 pipes of various diameters (from $12 \mathrm{~mm}$ to $50 \mathrm{~mm}$ ) form the CPS for one W7-X module as shown in Fig. 1. All those pipes need to be fixed into its positions properly in order to keep the stresses on an acceptable level, and to guarantee a collision free situation. In total 136 supports of different types are required for these purposes. Such a support carries from one up to six pipes and is fully fixed to either the magnet system (MS) or the outer vessel (OV). All pipes are grouped into several circuits corresponding to their special purposes, like feeder or return pipes for the coil cases, thermal insulation, etc. The pipe circuits of the coil casings, the support structure, and the thermal shields are connected to valves which regulate the coolant flow. The safety exhaust pipes are designed to reduce the pressure within the corresponding cooling loop in case of quench ("quench pipes").

\section{Analysis}

The main purposes of the analysis is to provide displacement vectors for the clash analysis, to define loads to be withstood by the pipe supports, and to check whether stresses in the pipes are within allowable limits. The task has to be carried out taking into account various assembly aspects, and with accurate consideration of numerous boundary conditions imposed by the interface and adjacent components, like the coils, structure elements, the current-leads, the outer-vessel, ports, thermal insulation, etc.

\subsection{Boundary conditions}

The boundary conditions for the CPS are rather complex, due to the fact that they come from numerous connected parts and necessary assumptions about the environment. To represent the CPS 
adequately it is necessary to consider the following (Fig. 2):

- cyclic symmetry at the module boundaries;

- displacements of the pipe connections at the outer vessel;

- displacements of the pipe connections at the current leads and coil headers;

- $\quad$ pressure forces on the quench pipes;

- displacements of the pipe connections at the structure and coils ;

- $\quad$ support clamps, mounted on the structure and coils;

- dead weight;

- thermal shrinkage;

- $\quad$ and internal pipe pressure.

The support clamps have to be considered as a special type of boundary conditions because they are subject of parametric studies at the same time. All other boundary conditions are principal boundary conditions which determine both the deformations and internal stresses in the system. The CPS has no significant backlash to these parts or environment conditions.

\subsection{Supports}

The supports (see Fig. 3) which keep the pipes in their positions and prevent them from clashes with other pipes or other nearby components, are vital parts of the CPS. In a first calculation assumption the supports are assumed to be pure axial hinges, allowing only the axial translation and rotation of the pipes. As a result the relative displacements of the coils introduce significant loads on the supports and high bending stresses within the pipes. The relative displacements are caused by the following effects:

- temperature difference between structural parts and the CPS during cool-down

- considerable deformation of the underlying structural components under electromagnetic 
forces (EM).

In order to reduce the forces and stresses obtained in the first run, additional degrees of freedom for the pipes have been introduced in the support clamps. They now allow also limited movements in perpendicular directions with regard to the pipe axis; this is realized by an annular gap between pipe and support clamp. Furthermore, the gap allows also rotations around all three axes within certain limits which leads to a self-adjusting CPS. As a result, the forces on the supports and the stresses in the pipes reduce significantly. The reduction is shown in Fig. 4, where the resulting forces and moments on two selected supports are plotted over the gap size. A gap of $1 \mathrm{~mm}$ size reduces the forces and moments dramatically. A further increase of the gap does not decrease the forces to a significantly lower level. This means that all constraints from the supports are released to a minimum, and the self adjustment of the CPS works fine. An additional benefit of the gap is that the assembly of the supports is eased due to reduced requirements concerning the accuracy for the pipes of installation.

\subsection{Flexible hoses and compensators}

Another crucial point for the CPS is the fact that some of the connected parts (e.g. valves, coil headers), cannot take any significant load. This means that even small forces with magnitudes of $20 \mathrm{~N}$ or less on these parts are not acceptable since they usually cause high bending stresses in the thin walls of the parts, or might destroy the component functionality. A kinematic decoupling of these parts is necessary. Since no space is available to lay out sufficient pipe loops for this purpose, the decoupling is realized by the use of flexible hoses and compensators. The flexible hoses are arranged twosome in a L-shaped configuration, as shown in Fig. 5 a, and placed nearby the critical component. The relative movements are simply compensated by them and do not result in any considerable constraints for the pipes. As a proof for this conclusion, a deformed Lshape bellow configuration is shown in Fig. $5 \mathrm{~b}$, and the components of the reaction forces at location $T$ in Table 1. An evaluation of the reaction forces at the transition from the pipe to the hoses shows that the loads are close to zero. The vertical component is the only relevant one because it represents the dead-weight force of the flexible hose. 
1.4.

\section{CPS reinforcements}

During the first iteration of the CPS analysis, the support and reinforcement structure was neither finalized nor fixed. The preliminary results showed immediately the weak points of the structure, which are the quench pipes (QP). They are directly loaded by the outer air pressure and cannot be decoupled from the CPS with flexible hoses or other flexible constructions. The analysis showed that it is strongly required to support these parts by a framework, keeping all QP together. This reduces the QP displacements and the resulting bending moments and stresses under operation.

\subsection{Clash analysis}

The exact prediction of displacements, like shown in Fig. 6, for the subsequent collision check is another major goal of the analysis. This check is vital to predict collisions, to elude damages and also contacts between parts at different temperatures, in order to avoid additional heat losses. Further, it is important for assembly personnel to know which parts need more attention and have to be assembled with smaller tolerances. A considerable number of collisions have been found after the first iteration; therefore, the routing of the affected pipes was significantly changed and used as input for the second iteration of the CPS which is under detail FEA analysis right now.

\section{Conclusions}

The cryogenic pipe system is a rather complex part of the W7-X cryostat due to many interfaces with other components. Its mechanical behaviour is mainly driven by the deformation of supporting structures, cool-down, and clamp design. The developed FE model is a powerful tool for predictions of CPS deformation, definition of loads on the cryopipe supports, and assessment of stresses in the pipes. The strong interference of the rather soft CPS with other components and its non-linear behaviour requires simulating the CPS with all its complex aspects. This approach finally allows providing important data for collision checks, design of the support, and assembly tolerances. 
Figures and tables (see below)

\section{Sizes:}

Fig. 1: 1/2 pp

Fig. 2: 1/6 pp

Fig. 3: 1/6 pp

Fig. 4: 1/6 pp

Fig. 5: 1/6 pp

Fig. 6: 1/2 pp

Fig. 1: Cryo-pipe system of W7-X: 1 - pipes, 2 - supports, 3 - valves, 4 - quench pipes, 5 - bellows

Fig. 2: Support boundary conditions for the CPS

Fig. 3: Typical pipe supports mounted on coil

Fig. 4: Reaction forces and moments vs. gap size at selected supports 1-ABE52HR430 and 1ABE52HR440

Fig. 5: L-shaped flexible hoses a) undeformed b) deformed under dead-weight and EM-loads

Fig. 6: Deformation of cryo-pipe system due to dead-weight, cooldown and EM loads on magnet system for the High-Jota load-case. The maximum ("MX") is $64 \mathrm{~mm}$, the minimum ("MN") $0 \mathrm{~mm}$.

Table 1: Typical reaction forces at transition from pipe to flexible hose (at location $\mathrm{T}$ in fig. 5) 


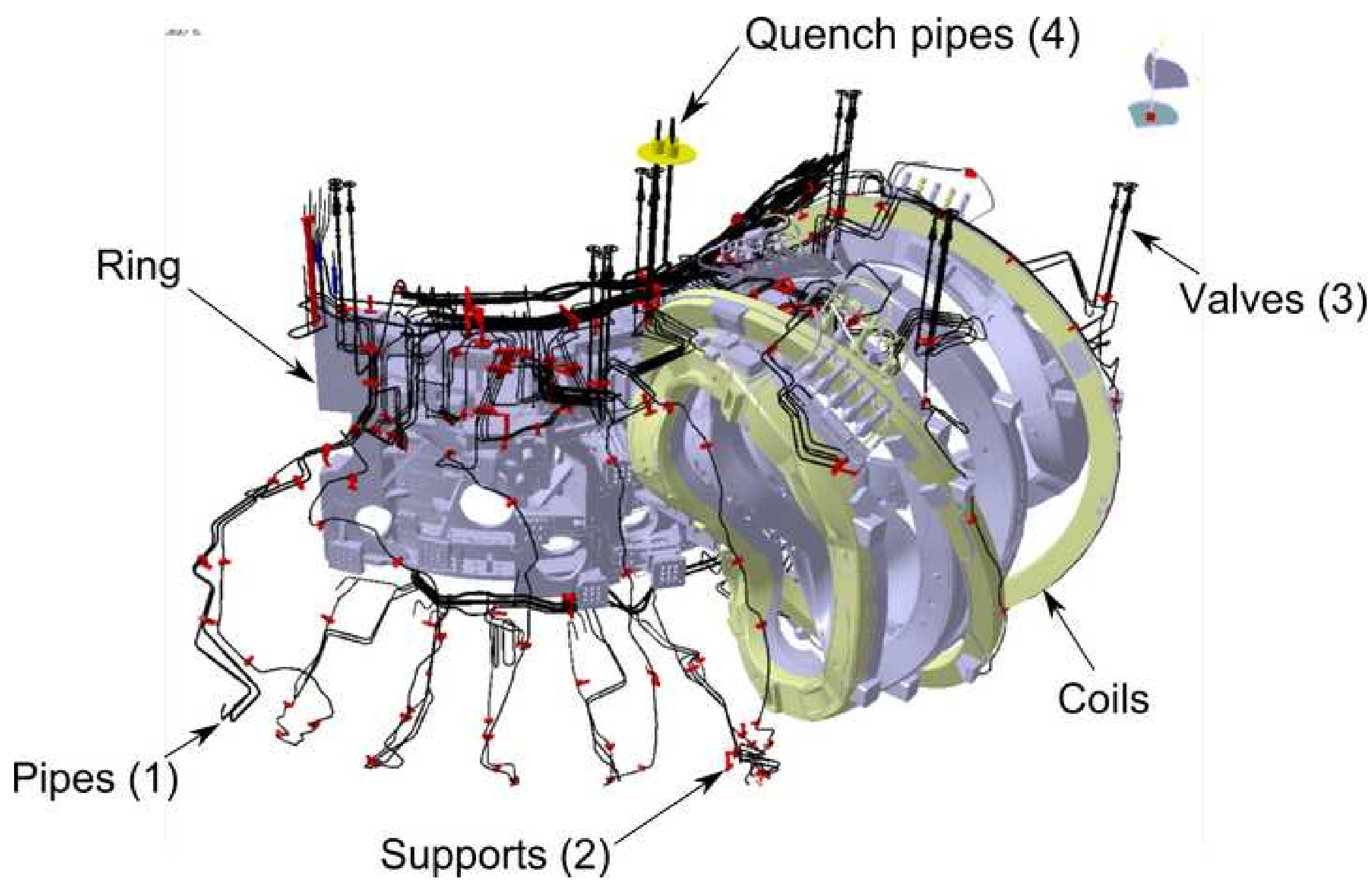




\section{Click here to download high resolution image}

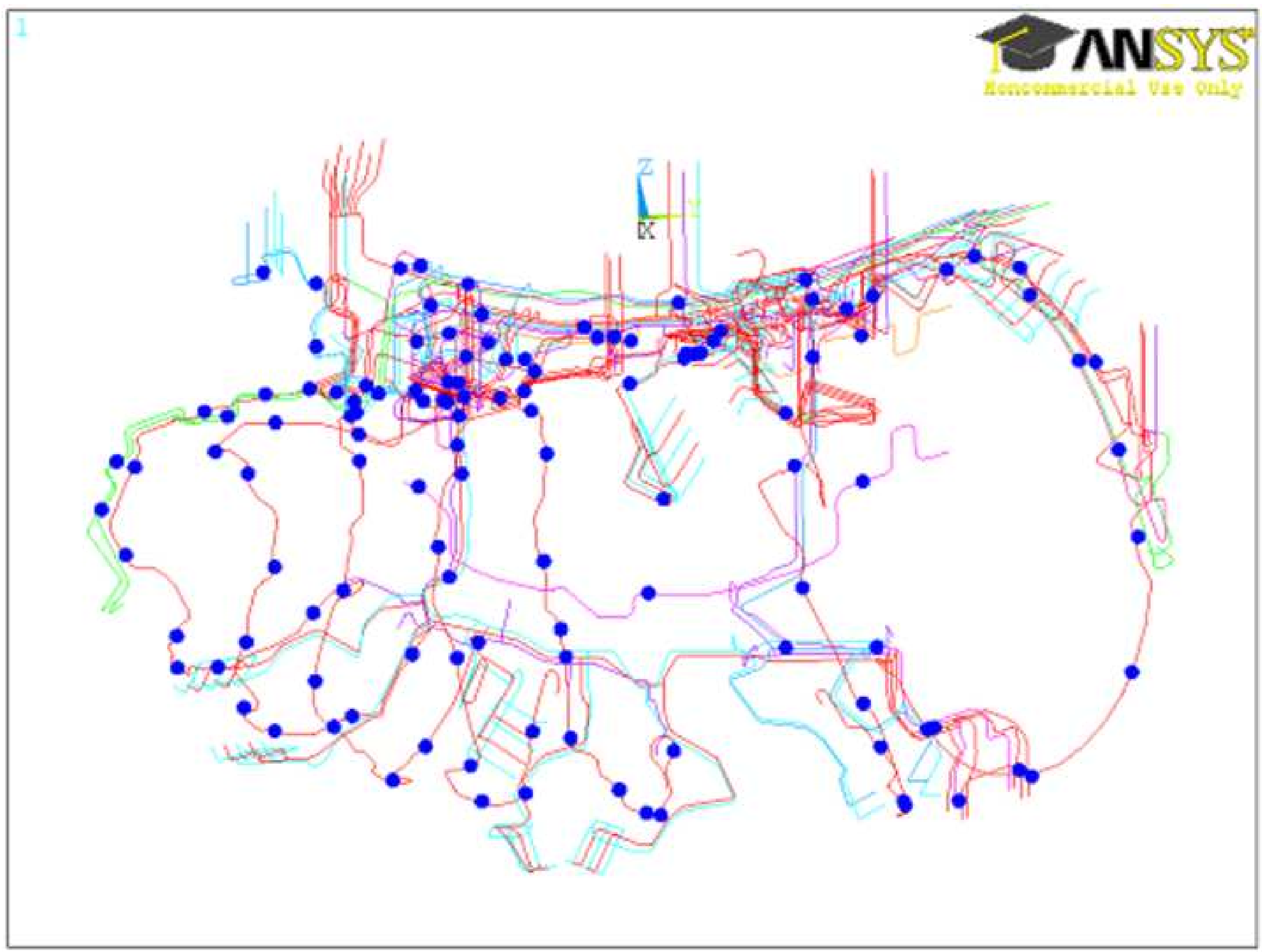


Typical pipe supports mounted on coil

Click here to download high resolution image
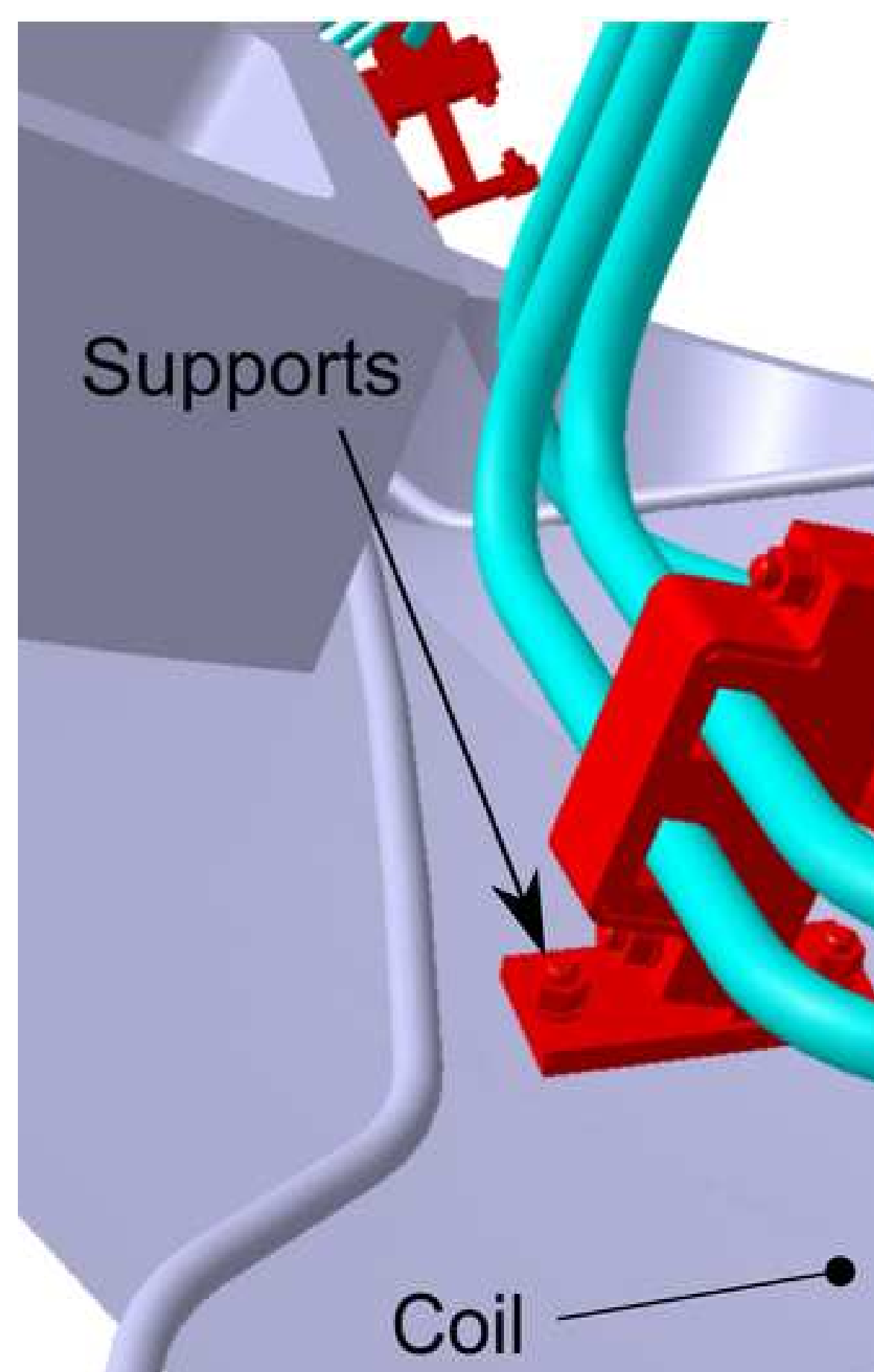

\section{Pipes}

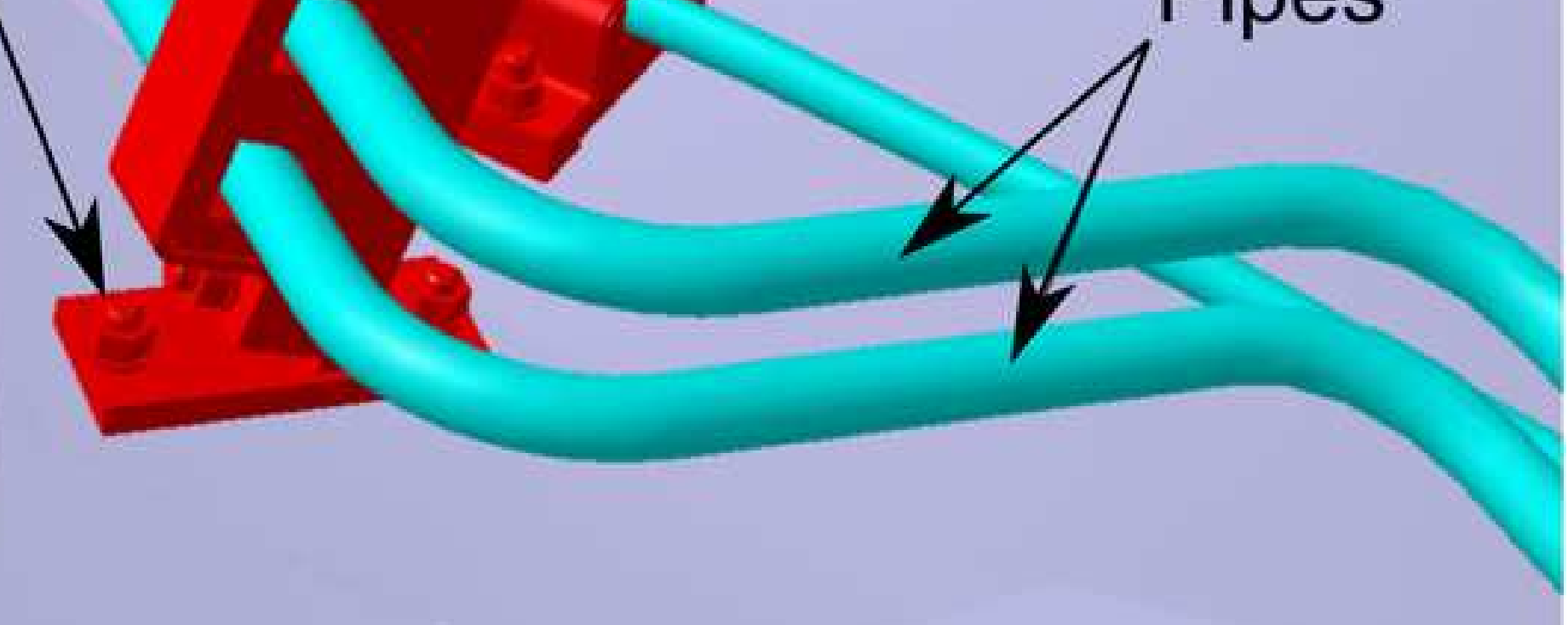




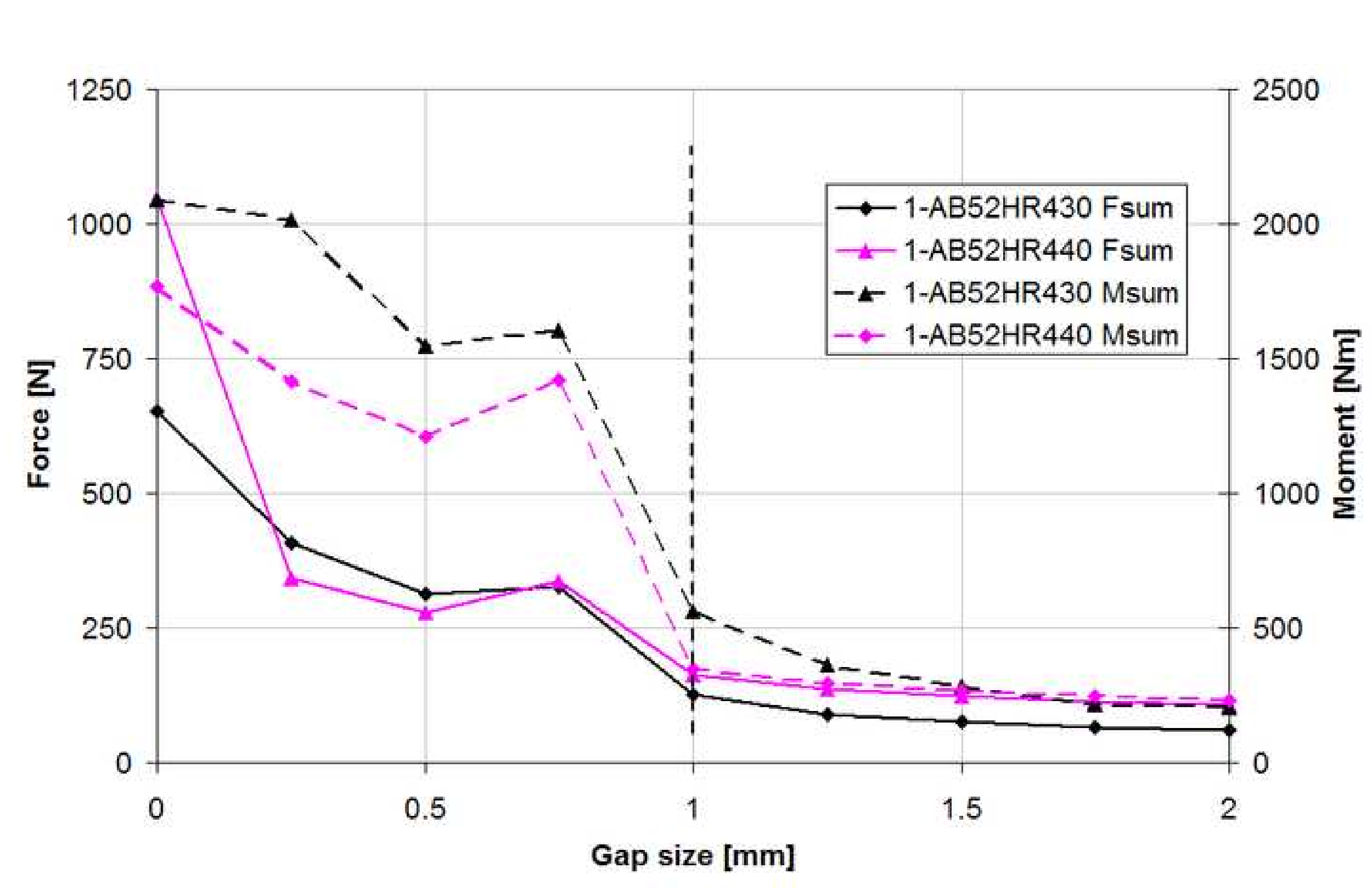

.

\begin{abstract}
C.ck
\end{abstract}




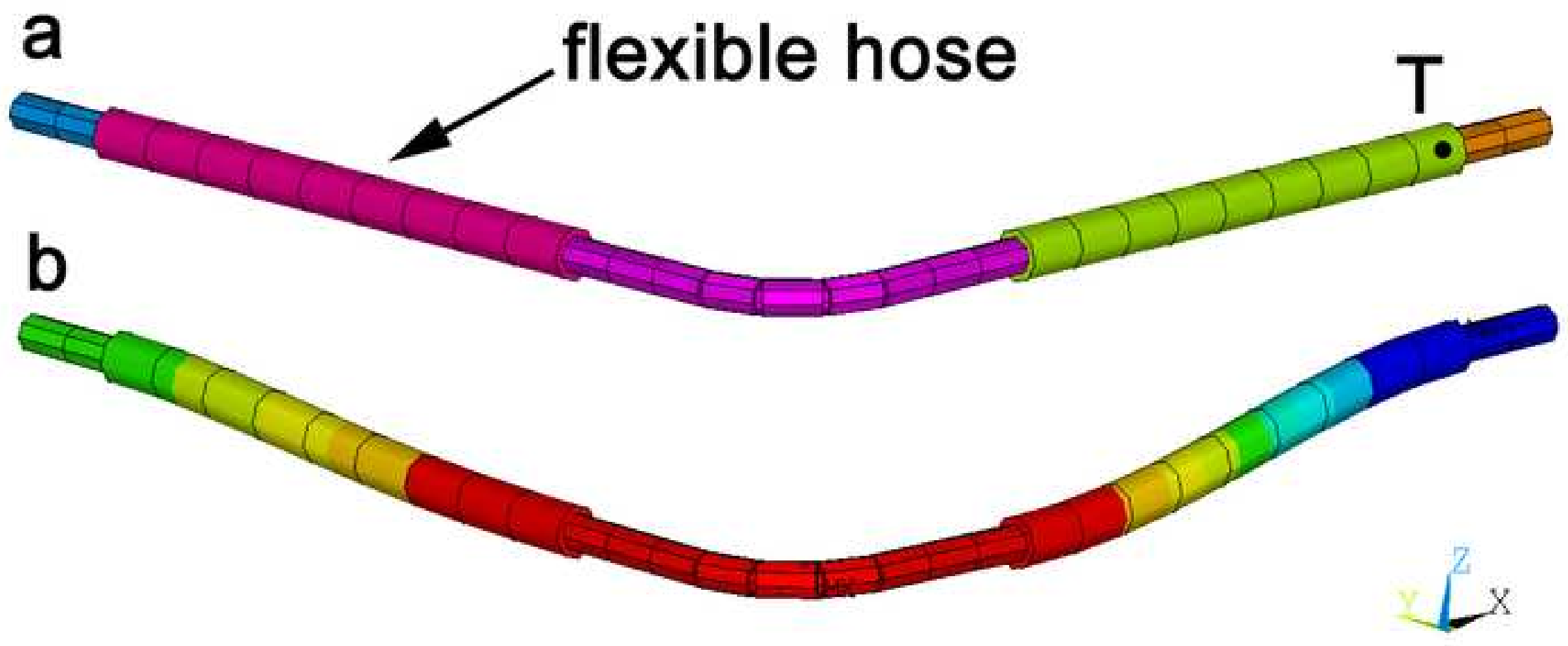


Deformation of cryo-pipe system

Click here to download high resolution image

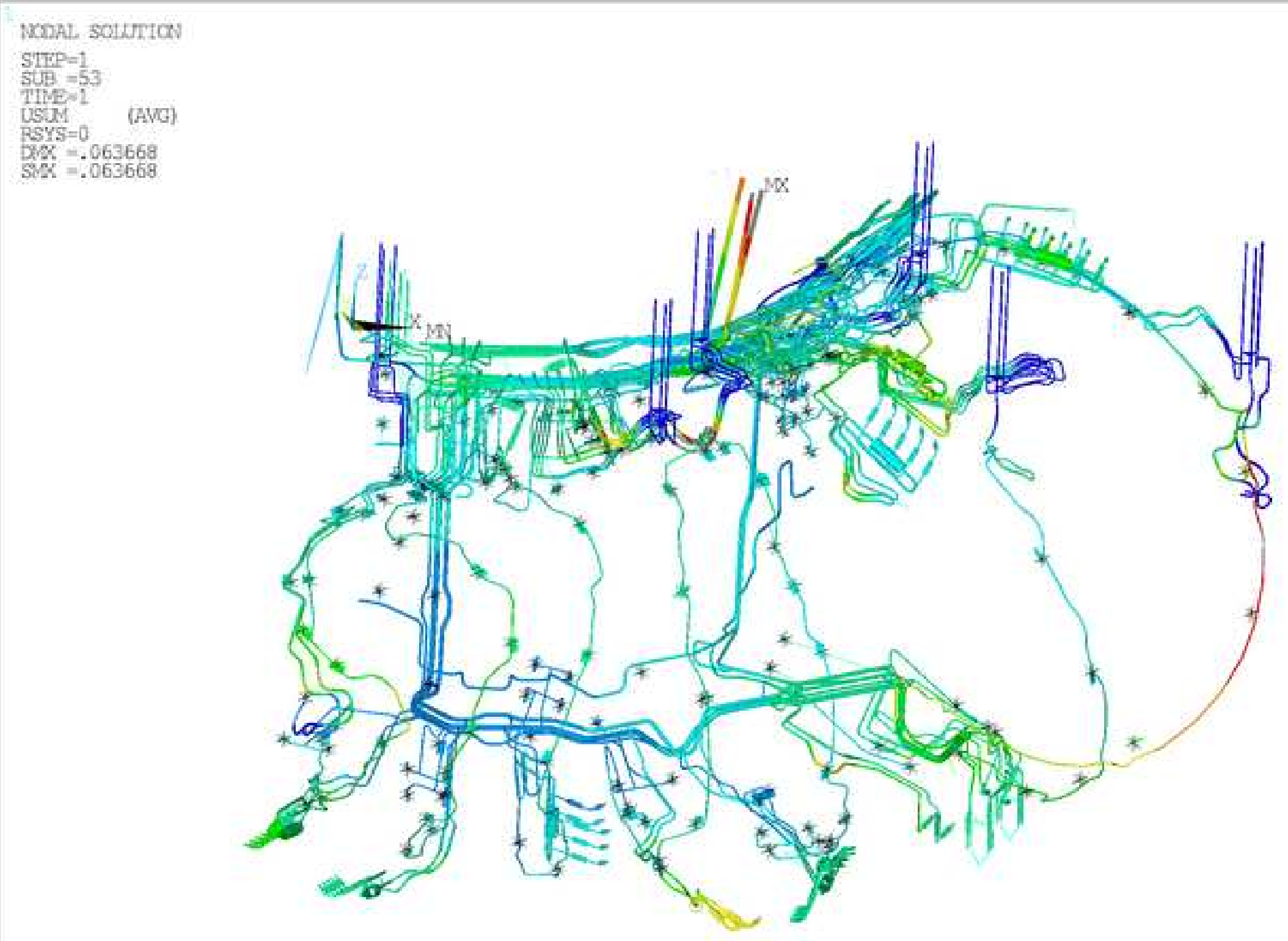

AWSYS 11.0SP1 $16: 58: 55$

PIOT NO. 
